\title{
Right Ventricular Outflow Tract Tachycardia Due To a Somatic Cell Mutation in G Protein Subunit ${ }_{\alpha i 2}$
}

\author{
Bruce B. Lerman, Bei Dong, Kenneth M. Stein, Steven M. Markowitz, Joel Linden, ${ }^{\ddagger}$ and Daniel F. Catanzaro* \\ Department of Medicine, Division of Cardiology, and $*$ Cardiovascular Center, The New York Hospital-Cornell Medical Center, New \\ York, 10021; and the ${ }^{\ddagger}$ Department of Medicine, Division of Cardiology, University of Virginia Health Science Center, Charlottesville, \\ Virginia 22908
}

\begin{abstract}
Idiopathic ventricular tachycardia is a generic term that describes the various forms of ventricular arrhythmias that occur in patients without structural heart disease and in the absence of the long QT syndrome. Many of these tachycardias are focal in origin, localize to the right ventricular outflow tract (RVOT), terminate in response to $\beta$ blockers, verapamil, vagal maneuvers, and adenosine, and are thought to result from cAMP-mediated triggered activity. DNA was prepared from biopsy samples obtained from myocardial tissue from a patient with adenosine-insensitive idiopathic ventricular tachycardia arising from the RVOT. Genomic sequences of the inhibitory $G$ protein $G_{\alpha i 2}$ were determined after amplification by PCR and subcloning. A point mutation (F200L) in the GTP binding domain of the inhibitory $G$ protein $\mathrm{G}_{\alpha \mathrm{i} 2}$ was identified in a biopsy sample from the arrhythmogenic focus. This mutation was shown to increase intracellular cAMP concentration and inhibit suppression of cAMP by adenosine. No mutations were detected in $\mathbf{G}_{\alpha \mathrm{i} 2}$ sequences from myocardial tissue sampled from regions remote from the origin of tachycardia, or from peripheral lymphocytes. These findings suggest that somatic cell mutations in the cAMP-dependent signal transduction pathway occurring during myocardial development may be responsible for some forms of idiopathic ventricular tachycardia. $(J$. Clin. Invest. 1998. 101:2862-2868.) Key words: G protein • ventricular tachycardia $\bullet$ signal transduction • arrhythmia
\end{abstract}

\section{Introduction}

Idiopathic ventricular tachycardia $(\mathrm{VT})^{1}$ is a generic term that describes various forms of VT that occur in patients without

Address correspondence to Bruce B. Lerman, Division of Cardiology, The New York Hospital-Cornell Medical Center, 525 East 68th Street, New York, NY 10021. Phone: 212-746-2169; FAX: 212-7468451; E-mail: blerman@mail.med.cornell.edu

Received for publication 22 August 1997 and accepted in revised form 7 April 1998

1. Abbreviations used in this paper: $\mathrm{AV}$, atrioventricular; CPA, cyclopentyladenosine; $\mathrm{G}_{\alpha \mathrm{i} 2}$, $\mathrm{G}$ protein subunit ${ }_{\alpha \mathrm{i} 2} ; \mathrm{hA}_{1} \mathrm{R}$, human adenosine receptor type 1 ; RVOT, right ventricular outflow tract; VT, ventricular tachycardia.

J. Clin. Invest.

(C) The American Society for Clinical Investigation, Inc. 0021-9738/98/06/2862/07 \$2.00

Volume 101, Number 12, June 1998, 2862-2868

http://www.jci.org structural heart disease (1). The most common form of idiopathic VT originates from the right ventricular outflow tract (RVOT), is adrenergically mediated, and has characteristics that are consistent with cAMP-mediated triggered activity due to delayed afterdepolarizations. This form of tachycardia typically terminates in response to perturbations that lower stimulated levels of intracellular calcium, i. e., adenosine, verapamil, and enhanced vagal tone (2-4). Termination of idiopathic VT in response to adenosine is thought to be diagnostic of a cAMP-dependent mechanism since the ventricular electrophysiologic effects of adenosine are mediated through the inhibitory $G$ protein $G_{i}$, which inhibits adenylyl cyclase and decreases intracellular cAMP (5-7).

A patient with adrenergically mediated idiopathic RVOT VT that was unresponsive to adenosine and vagal maneuvers was identified. Because of insensitivity of the tachycardia to adenosine, we investigated the possibility that a mutation in the inhibitory branch of the cAMP signal transduction pathway could have elevated intracellular cAMP and facilitated the spontaneous initiation of VT. In the present study, we identified a point mutation in the $\mathrm{G}_{\alpha \mathrm{i} 2}$ gene from the arrhythmogenic focus of this patient. Both stable and transient transfection of CHO-K1 cells with the mutant $\mathrm{G}_{\alpha \mathrm{i} 2}$ showed that this mutation increases stimulated levels of cAMP and prevents adenosine suppression of cAMP.

\section{Methods}

Clinical data. The subject is a 58-yr-old man who developed sustained monomorphic VT during an intense argument. The tachycardia was associated with chest pain, dyspnea, diaphoresis, and a systolic blood pressure of $60 \mathrm{mmHg}$. The cycle length of tachycardia was $240 \mathrm{~ms}$. Emergency medical services restored sinus rhythm with a 200 J shock. The resting ECG was normal, as was an echocardiogram. Cardiac catheterization demonstrated normal coronary anatomy and normal left ventricular function. There was no family history of ventricular tachycardia or sudden cardiac death.

Electrophysiologic study. An electrophysiologic study was performed with the patient in the unsedated postabsorptive state after informed consent was obtained. Percutaneous endocardial biopsies were obtained with a bioptome from the right ventricle after informed consent was obtained for a protocol approved by the Human Investigations Committee. Three quadripolar electrode catheters were inserted percutaneously and advanced under fluoroscopic guidance to the high right atrium, right ventricular apex, and atrioventricular (AV) junction for recording of the His-bundle electrogram. Bipolar intracardiac recordings were filtered at $40-400 \mathrm{~Hz}$ and were displayed simultaneously with three surface ECG leads on a multichannel oscilloscope. Stimulation was performed with a programmable stimulator and an isolated constant current source (Bloom Associates, Reading, PA). Stimuli were delivered as rectangular pulses of 2-ms duration at four times the diastolic threshold.

The stimulation protocol included the introduction of single, double, and triple extrastimuli during several paced cycle lengths from the high right atrium, right ventricular apex, and RVOT. In addition, 
sequential atrial and ventricular burst pacing for 15-30 beats was performed, beginning at a cycle length of $500 \mathrm{~ms}$ and decreasing in 10-ms steps to $250 \mathrm{~ms}$ (or to pacing-induced AV nodal Wenckebach in the case of atrial pacing). The ability of isoproterenol ( $4 \mu \mathrm{g} / \mathrm{min})$ to initiate VT alone, during its washout phase, and during concurrent pacing was also evaluated (Fig. 1).

$D N A$ preparation, PCR amplification, and sequencing. Three biopsy samples were obtained from the site of origin of ventricular tachycardia (RVOT) and two from the inferior region of the right ventricular septum. After excision, one sample from the RVOT was processed for routine histological examination, and the remaining biopsy specimens were frozen rapidly and stored for molecular analysis. To prepare genomic DNA, biopsies were thawed in $100 \mu \mathrm{l}$ lysis buffer $(150 \mathrm{mmol} /$ liter $\mathrm{NaCl}, 10 \mathrm{mmol} /$ liter Tris $\cdot \mathrm{Cl}[\mathrm{pH} 8.3]), 10$ $\mathrm{mmol} /$ liter EDTA, and $0.4 \%$ sodium dodecyl sulfate) containing 0.1 $\mathrm{mg} / \mathrm{ml}$ proteinase $\mathrm{K}$ and incubated for 12 to $24 \mathrm{~h}$ at $50^{\circ} \mathrm{C}$. Proteinase $\mathrm{K}$ was heat-inactivated at $98^{\circ} \mathrm{C}$ for $3 \mathrm{~min}$. DNA was extracted with phenol and chloroform, ethanol precipitated, and then dissolved in $50 \mu \mathrm{l}$ of $10 \mathrm{mmol} / \mathrm{liter}$ Tris $\mathrm{Cl}(\mathrm{pH} 8.0), 1 \mathrm{mmol} /$ liter EDTA. Samples were diluted 1:10 in PCR buffer (Perkin-Elmer Cetus, Emeryville, CA). The strategy for PCR amplification is shown in Fig. 2 A. Primers A-D were originally described by Lyons et al. (8). Primer E corresponds to part of the sequence in exon 7. The sequence of each primer was as follows: $(A) 5^{\prime}$-CCCCCCATCCCCAGCTACCT-3'; (B) 5'-TCTCACCATCTCCTCGTCCTC-3'; (C) 5'-ATTGCACAGAGTGACTACATCCCC-3'; $(D)$ 5'-GGCGCTCAAGGCTACGCAGAA-3'; (E) 5'-GAA TAG CTT CAT GCT CTC ATG C-3'. PCR products were cloned into the pCRII vector (Invitrogen Corp., San Diego, CA) and sequenced as described previously (4).

Functional analysis of the mutation. The F200L mutation of $\mathrm{G}_{\alpha \mathrm{i} 2}$ identified in genomic samples was introduced into the full-length $G_{\alpha i 2}$ cDNA using the PCR overlap method (9). Both the mutated cDNA, and its counterpart containing the native sequence, were separately inserted into the expression vector pCEP4. These constructions were individually transfected into CHO-K1 cells, either native cells, or cells that had been stably transfected with the myocardial human $\mathrm{A}_{1}$ adenosine receptor $\left(\mathrm{hA}_{1} \mathrm{R}\right)(10)$. Cells were grown in Ham's F12 medium containing $10 \%$ fetal bovine serum at $37^{\circ} \mathrm{C}$ in a humidified atmosphere containing $5 \% \mathrm{CO}_{2}$. Cells containing the stably transfected pCEP4 constructs were selected on the basis of hygromycin resis- tance. Cells were isolated using cloning cylinders and clones were then established by plating at limiting dilution.

Transient transfection experiments were also carried out using the same $\mathrm{G}_{\alpha \mathrm{i} 2}$ constructs and cells described above. In these experiments $\sim 10^{6}$ cells were transfected with a calcium phosphate precipitate containing $20 \mu \mathrm{g}$ DNA (11). After $48 \mathrm{~h}$ cells were treated with forskolin and cyclopentyladenosine (CPA) as described below.

To assess the effects of forskolin and CPA, cells were placed in serum-free medium for $2 \mathrm{~h}$ at $37^{\circ} \mathrm{C}$. The medium was then replaced with serum-free medium containing either vehicle, forskolin, or forskolin $+\mathrm{CPA}$, and the incubation continued at $37^{\circ} \mathrm{C}$ for $10 \mathrm{~min}$. cAMP levels were determined by radioimmunoassay (12).

Statistical methods. Results are presented as mean \pm SEM. Data for transfection experiments were analyzed by a factorial ANOVA. Multiple comparisons between treatments and between groups were assessed for significance by the Sheffe $F$ test. For all comparisons, a probability value $P<0.05$ was required to reject the null hypothesis.

\section{Results}

Electrophysiologic findings. Sinus node, atrial, and His-Purkinje system function were all normal. Dual AV nodal pathways were present, but AV nodal reentrant tachycardia was not inducible with up to double atrial extrastimuli. In the resting state, VT was not inducible with up to triple ventricular extrastimuli introduced from two right ventricular sites (4). However, sustained VT was induced reproducibly with a single ventricular extrastimulus coupled to an 8-beat drive during concurrent infusion of isoproterenol ( $4 \mu \mathrm{g} / \mathrm{min}$ i.v.). The morphology of tachycardia was left bundle branch block with a left inferior axis and the cycle length of tachycardia was 210-220 ms (Fig. 1). The site of origin of tachycardia was localized to the RVOT by activation and pace mapping (3). The tachycardia was reproducibly terminated with rapid ventricular pacing. Adenosine (150-1,000 $\mu \mathrm{g} / \mathrm{kg}$ i.v. bolus), Valsalva, carotid sinus massage, and lidocaine (100 mg i.v.) had no effect on tachycardia. Histology of the RVOT biopsy sample did not demonstrate fibrous tissue or fat deposition.
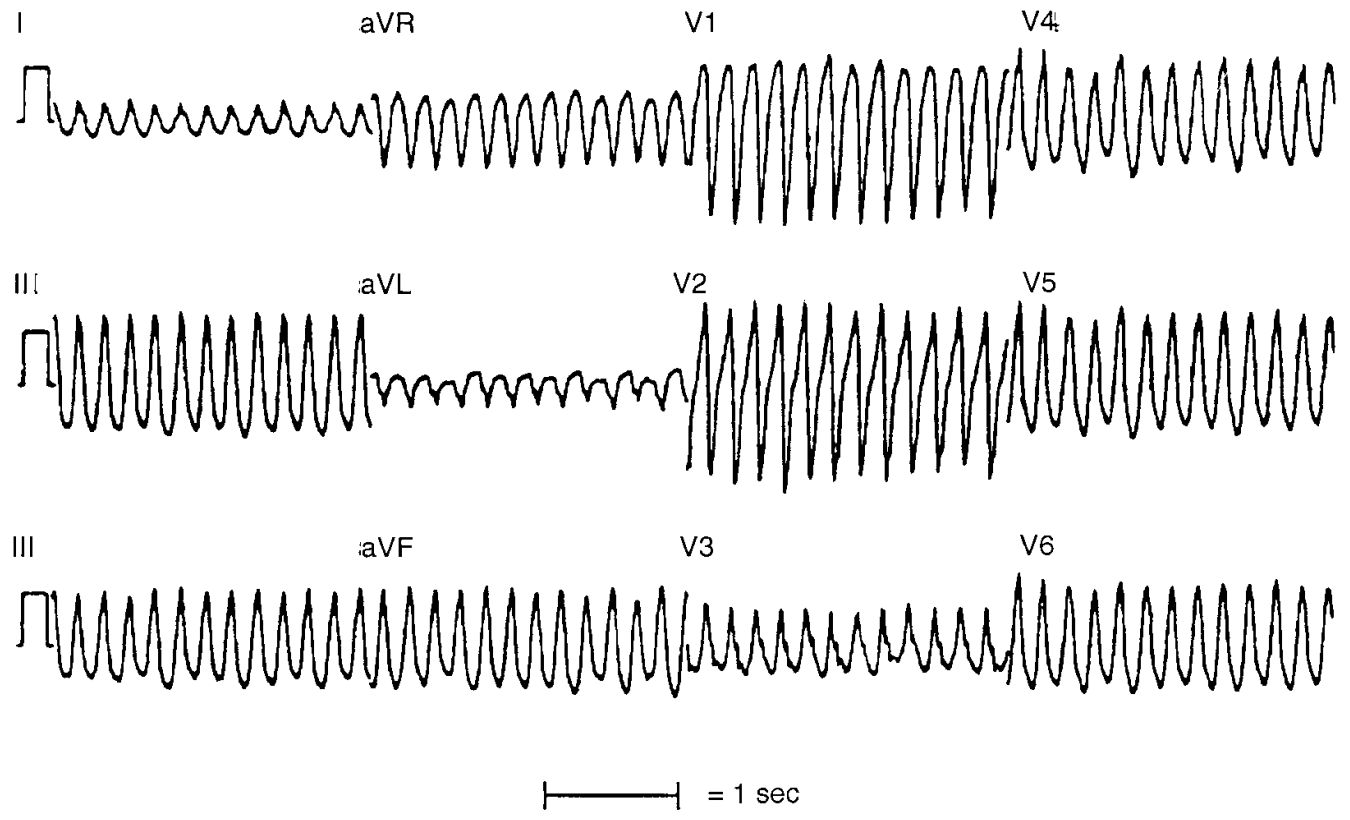

Figure 1. Twelve-lead ECG of inducible ventricular tachycardia. 


\section{A}

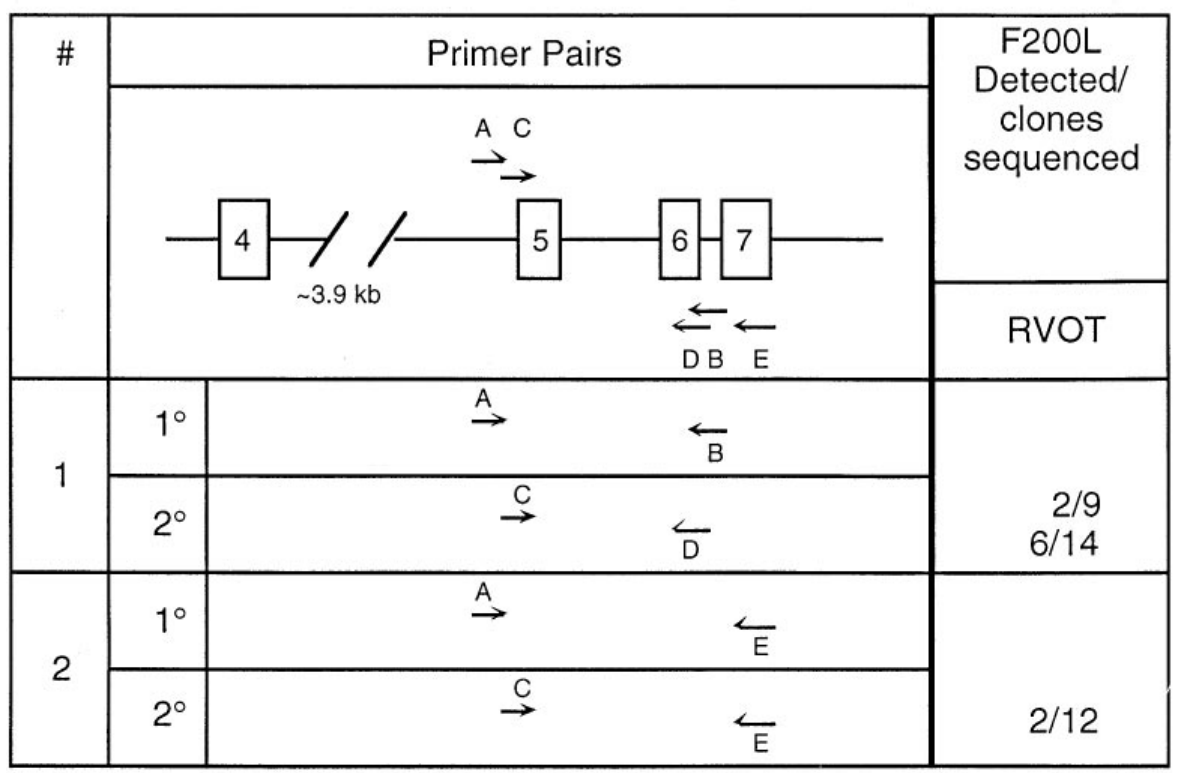

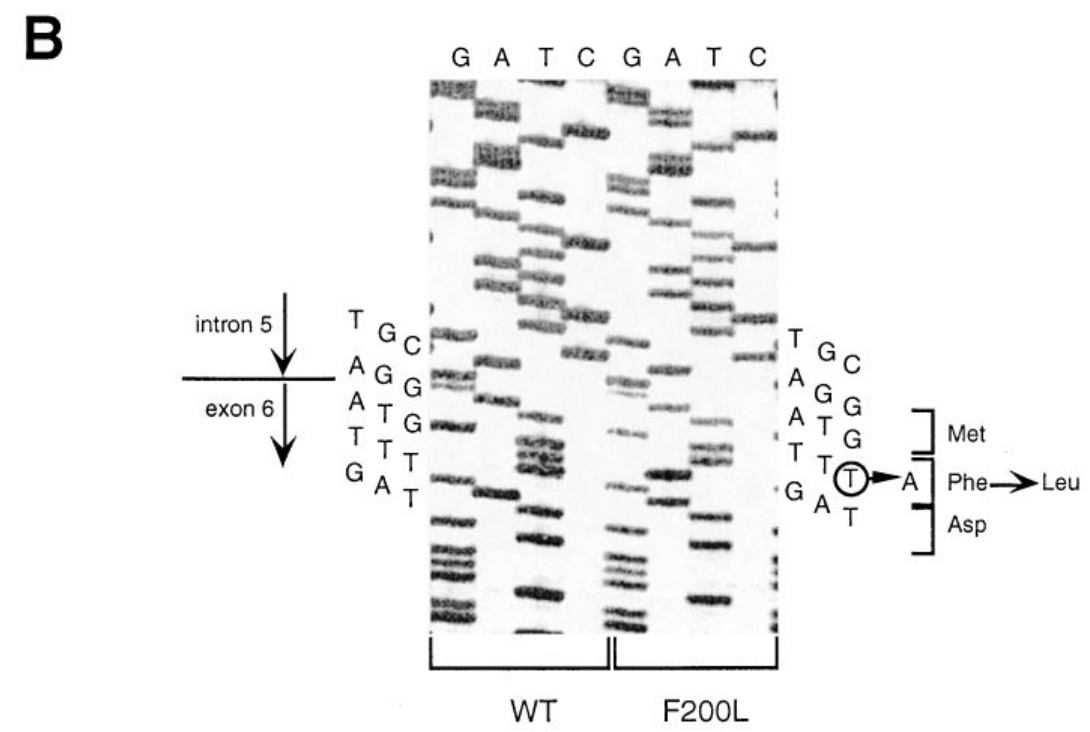

Figure 2. PCR amplification strategy employed for the detection and verification of the F200L mutation. $(A)$ Regions of $\mathrm{G}_{\alpha \mathrm{i} 2}$ amplified and location of primers used (9). Numbered boxes indicate exons and horizontal lines indicate intervening sequences. Shown are the strategies for two separate PCRs indicated in the left-hand column. For each strategy the primer pairs used for the primary and secondary amplification are indicated. Arrows indicate PCR primers. (B) Representative sequencing of native and F200L mutant clones.

The patient was subsequently treated with radiofrequency catheter ablation of the arrhythmogenic focus (13). Due to the fact that this form of RVOT VT represented a unique type of tachycardia and because of its associated hemodynamic compromise, we also elected to implant an internal defibrillator. During the 4-yr follow-up period, there has been no recurrence of VT or device discharge, suggesting that the arrhythmogenic focus was successfully ablated.

Genetic analysis. The myocardial adenosine $\mathrm{A}_{1}$ receptor is coupled to adenylyl cyclase through $\mathrm{G}_{\alpha \mathrm{i} 2}$. Since mutations in the GTP-binding domain of various $G$ proteins have been shown to result in both constitutive activation and inactivation (14), we sequenced this region of $\mathrm{G}_{\text {oi2 }}$ (encoded in exons 5 and 6). DNA from biopsy samples was amplified by PCR (Fig. 2A) and either sequenced directly or cloned into the pCRII vector. Direct sequencing of this region in DNA from samples obtained from the site of origin of ventricular tachycardia in the RVOT revealed a sequence that agreed with the published sequence (15) except for one region in which a faint additional band was observed corresponding to an additional $\mathrm{A}$ base at the third codon position of residue 200 (not shown). Sequence analysis of two separately cloned pools revealed that $2 / 9$ and 
$6 / 14$ clones contained this mutation (TTT $\rightarrow$ TTA), which converted the residue encoded at this position from phenylalanine to leucine (F200L) (Fig. 2 B).

In the original PCR, primer pair $\mathrm{A}+\mathrm{B}$ was used for the primary amplification that was then reamplified with primer pair $\mathrm{C}+\mathrm{D}$. In subsequent analyses, primer pairs $\mathrm{A}+\mathrm{E}$ and $\mathrm{C}+\mathrm{E}$ were used in a nested PCR. The F200L mutation was detected in 2/12 clones that were isolated and sequenced. Using this latter strategy, it is highly unlikely that a contaminant arising from any combination of the original primers A, B, C, and $\mathrm{D}$ would have been reamplified. No mutations were found upon reamplification of DNA from right ventricular septal biopsy samples obtained at sites remote from the origin of VT $(0 / 7,0 / 7)$, or from peripheral lymphocyte DNA (0/12). Furthermore, no mutations were detected in any other coding region of $\mathrm{G}_{\alpha \mathrm{i} 2}$ in DNA from the RVOT biopsy in at least ten separate clones analyzed for each exon of $\mathrm{G}_{\alpha \mathrm{i} 2}$ (data not shown).

Functional studies of the F200L mutation $G_{\alpha i 2}$. To evaluate the functional effect of F200L, this mutation was introduced into the sequence of the full-length $\mathrm{G}_{\mathrm{\alpha i} 2}$ contained in the expression vector $\mathrm{pCEP} 4$. This construct, or its counterpart containing the native sequence, was stably transfected into $\mathrm{CHO}-$ $\mathrm{K} 1$ cells, either the native cells, or ones that had previously been stably transfected to express the myocardial $\mathrm{hA}_{1} \mathrm{R}$ (10). We isolated five separate clones of the native and F200L mutant constructs in the CHO-K1 cells. Densitometry of Western blots probed with antibodies specific for $\mathrm{G}_{\alpha \mathrm{i}}$ revealed that the levels of $\mathrm{G}_{\alpha \mathrm{i}}$ in the transfected clones were approximately twofold greater than the untransfected cells, and differed by $<25 \%$ between clones. Responses of cAMP levels to forskolin and CPA were tested in three separate clones for each construct. Each experiment was carried out three times and the results of one experiment are shown in Fig. 3. In cells transfected with the native construct, forskolin significantly increased cAMP levels $16 \pm 3$-fold, an effect that was suppressed to near basal levels by the addition of CPA. In cells transfected with the F200L construct, forskolin also increased cAMP levels (21 $\pm 1-$ fold, $P=0.0002$ ), but under these conditions CPA no longer suppressed cAMP (Fig. 3). The increase in cAMP levels induced by forskolin were also significantly higher in cells transfected with the mutant construct than in cells transfected with its native counterpart $(P=0.003)$, suggesting that the mutation may also increase the stimulated levels of cAMP.

To further explore the effects of the F200L mutation, transient transfection experiments were performed to determine the dose-response relationship between the amount of mutant $\mathrm{G}$ protein expressed and the response of cAMP to forskolin stimulation (Fig. 4). In these experiments $\mathrm{CHO}$ cells, stably transfected with $\mathrm{hA}_{1} \mathrm{R}$, were transiently transfected with progressively increasing amounts of the F200L construct. Since changing the intracellular concentration of the $G$ protein might affect the cellular response to forskolin and/or adenosine, the proportion of native:mutant $\mathrm{G}$ protein construct was varied while maintaining a constant amount of the transfected $\mathrm{G}$ protein construct. In the absence of $\mathrm{hA}_{1} \mathrm{R}$, there was no suppression by CPA and no effect of the F200L mutation (not shown). In $h A_{1} R$ cells untransfected with $G_{\alpha i 2}$, cAMP levels were slightly lower $(32 \%)$ than in cells transfected with native $\mathrm{G}_{\alpha \mathrm{i} 2}$ alone $(P=\mathrm{NS})$. This could be due to constitutive activity of the recombinant receptors or partial receptor activation by endogenous adenosine. Activation of receptors coupled to the inhibition of adenylyl cyclase is known to trigger a compensa- tory increase in adenylyl cyclase activity (16-18), resulting in an augmented production of cAMP in response to forskolin. Similarly, overexpression of the native inhibitory $G$ protein and $\mathrm{hA}_{1} \mathrm{R}$ could increase forskolin-stimulated adenylyl cyclase activity.

In cells transfected with the $\mathrm{G}_{\mathrm{\alpha i} 2}$ constructs, differences in cAMP levels between varying ratios of native/F200L were not significant for the control treament, but highly significant for both the forskolin - and forskolin + CPA treatments $(P<$ 0.0001, Fig. 4). In particular, the increment in cAMP in response to forskolin was linearly related to the amount of mutant $\mathrm{G}_{\alpha \mathrm{i} 2}$ transfected $\left(r^{2}=0.898, P<0.0001\right)$. These results are consistent with our findings using stably transfected cells (Fig. 3) and confirm that the mutant G protein has the ability to increase the stimulated levels of intracellular cAMP. Suppression of forskolin-mediated stimulation of cAMP by CPA remained relatively constant until excess mutant $G_{\alpha i 2}$ was cotransfected, at which point the effect of CPA was abolished. When equal amounts of native and F200L $\mathrm{G}_{\alpha \mathrm{i} 2}$ were cotransfected, the relative fall in cAMP concentration secondary to CPA was the same as in cells transfected with native $G_{\alpha i 2}$ alone; however, the absolute cAMP concentration was greater in cells cotransfected with the mutant $\mathrm{G}_{\mathrm{\alpha i2}}$ (Fig. 4).

In separate transfection experiments using an RSV $\beta$-gal expression plasmid we estimated the $\mathrm{CHO}$ cells to be transfected at a frequency of $15-27 \%$ (not shown). Because only this subset of cells can express the transfected constructs, the elevation of forskolin stimulated cAMP levels by mutant $\mathrm{G}_{\alpha \mathrm{i} 2}$

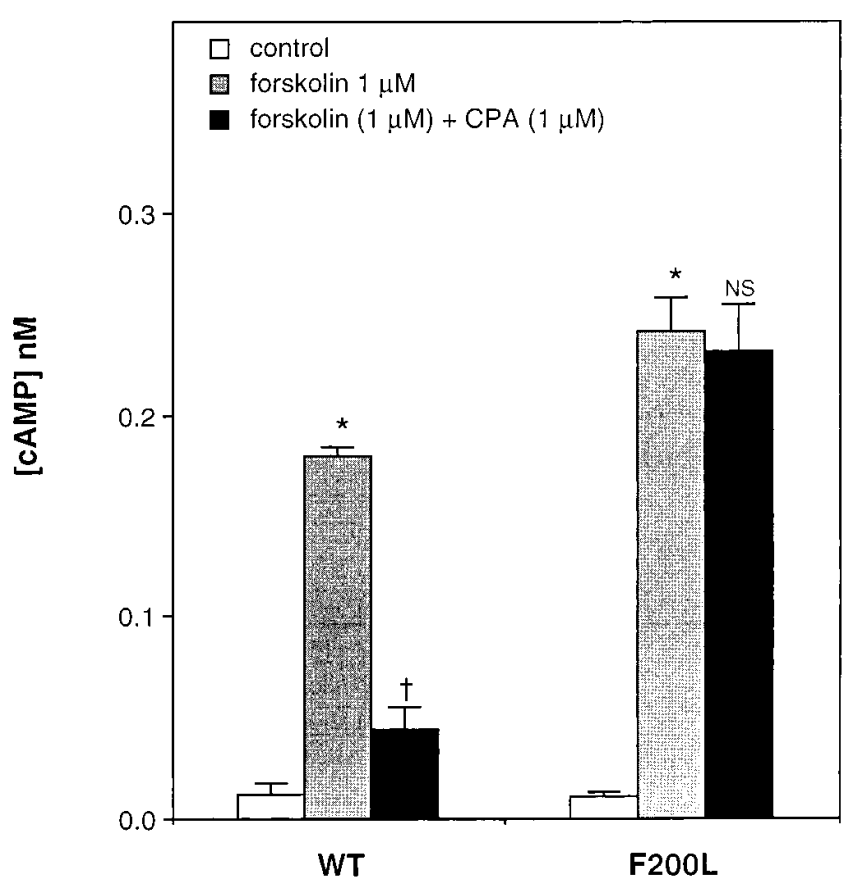

Figure 3. Effects of forskolin and cyclopentyladenosine (CPA) on cAMP levels in $\mathrm{CHO}$ cells stably transfected with human adenosine $\mathrm{A}_{1}\left(\mathrm{hA} \mathrm{A}_{1} \mathrm{R}\right)$ receptor (10) and either native (WT) and mutant (F200L) $\mathrm{G}_{\text {ai2 }}$. Differences in cAMP levels between control and forskolin were significant at $P<0.0001$ for both WT- and F200L-transfected cells $(*)$. Differences in cAMP levels between forskolin and forskolin + CPA were significant at $P<0.0001(\dagger)$ for WT-transfected cells, but not significant $(N S)$ for F200L-transfected cells. 


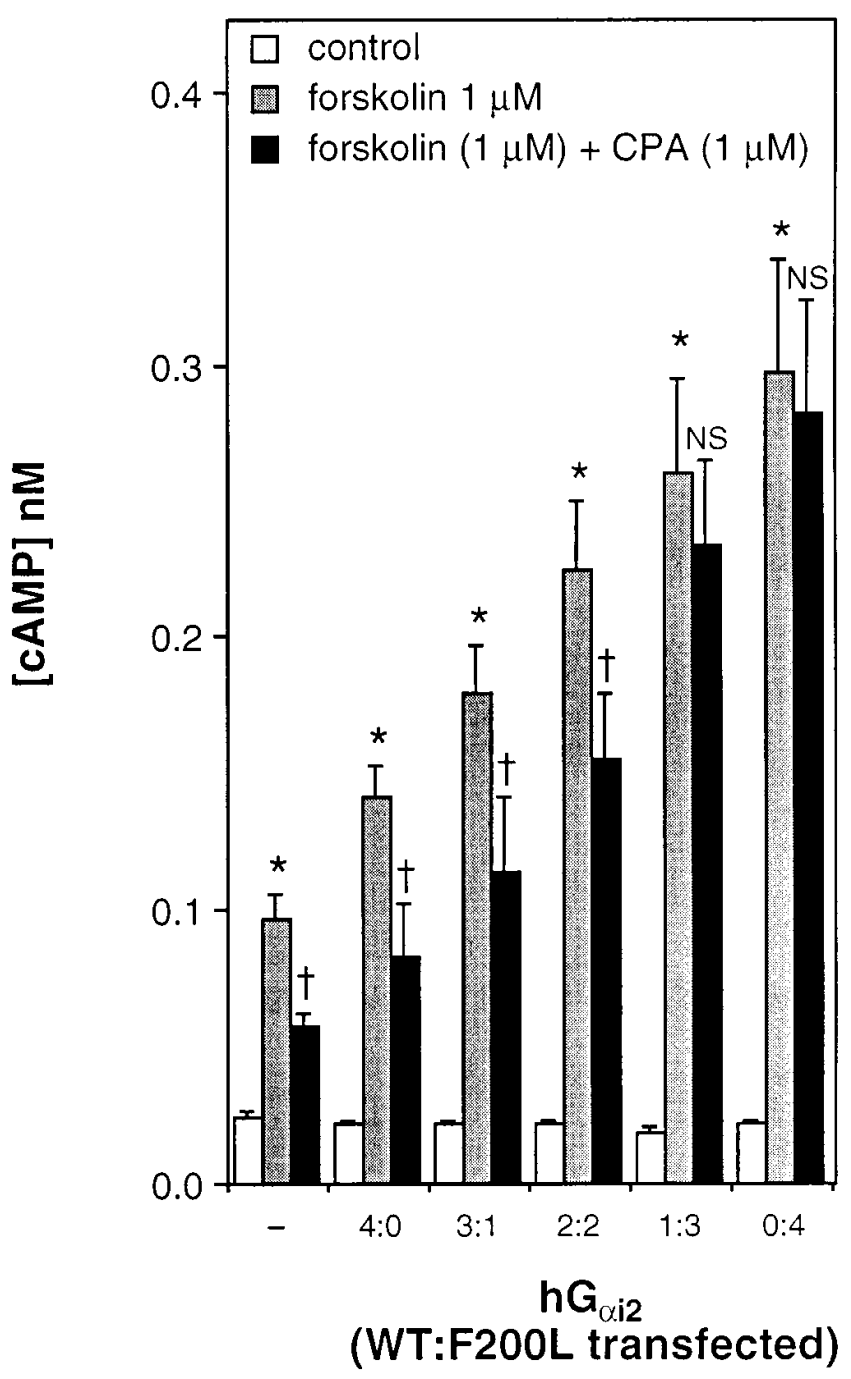

Figure 4. Effects of forskolin and cyclopentyladenosine (CPA) on cAMP levels in $\mathrm{CHO}$ cells transiently transfected with varying ratios of native $(W T)$ and mutant $(F 200 L) \mathrm{G}_{\mathrm{\alpha i} 2}$ in the presence of stably transfected human adenosine $\mathrm{A}_{1}$ receptor $\left(\mathrm{hA}_{1} \mathrm{R}\right)$. Differences in cAMP levels between control and forskolin were significant at $P<$ $0.005(*)$ for all transfection conditions. Differences in cAMP levels between and forskolin and forskolin $+\mathrm{CPA}$ were significant at $P<$ 0.05 for transfection conditions marked $\dagger$, or not significant $(N S)$.

would mask adenosine suppression mediated by the endogenous native $\mathrm{G}_{\alpha \mathrm{i} 2}$. This would suggest that most of the forskolinstimulated cAMP is derived from transfected cells.

Taken together, these observations suggest that the F200L mutation results in inactivation of $\mathrm{G}_{\alpha \mathrm{i} 2}$ leading to the loss of the inhibitory effect of adenosine. In the heart, the mutation may also increase basal and catecholamine-stimulated cAMP accumulation. These changes are analogous to the effects of pertussis toxin on $\mathrm{G}_{\alpha \mathrm{i}}$, which also results in both the loss of inhibitory modulatory effects and an increase in forskolin-stimulated cAMP $(19,20)$.

\section{Discussion}

The principal finding of this study is the identification of a focal somatic myocardial mutation in $\mathrm{G}_{\alpha \mathrm{i} 2}$ whose phenotypic ex- pression is consistent with the patient's idiopathic ventricular tachycardia. The spontaneous initiation of adrenergically mediated VT in this patient could have been potentiated by the mutation in several ways. The F200L mutation in human $\mathrm{G}_{\alpha \mathrm{i} 2}$ may disrupt adenosine signaling and thereby abolish the antiadrenergic (and thus antiarrhythmic) effects of endogenous adenosine, leading to increased intracellular cAMP $(21,22)$. In addition, the mutation may increase the concentration of intracellular cAMP in response to $\beta$-adrenergic stimulation independently of its effects on adenosine.

The tachycardia in this patient had features in common with the usual form of idiopathic VT. The absence of structural heart disease in this patient was confirmed by a normal ECG and results from echocardiography, cardiac catheterization, and myocardial biopsy. The tachycardia was dependent on catecholamine stimulation and had a focal site of origin in the RVOT. However, the tachycardia also had several unique characteristics. Although adrenergically mediated, it was unresponsive to adenosine, a finding that led us to examine adenosine signaling and $\mathrm{G}$ protein function. The tachycardia also failed to respond to vagal maneuvers, a finding consistent with the $G$ protein mutation we identified. An automatic mechanism was excluded since programmed stimulation reproducibly initiated and terminated tachycardia. These data are therefore most consistent with adrenergically mediated RVOT tachycardia due to triggered activity or reentry.

In transient transfection experiments, the stimulated levels of cAMP increased in proportion to amount of mutant G protein transfected. Therefore, although CPA suppressed cAMP levels at transfection ratios 3:1 and 2:2 (native/mutant), the absolute level of cAMP in cells transfected at the 2:2 ratio in response to forskolin plus CPA was the same, if not slightly higher, than in cells transfected with only native $G$ protein and treated with forskolin alone. While it is difficult to infer a mechanism for the arrhythmia from the artificial setting of this experiment in which both $\mathrm{G}$ proteins and receptors are overexpressed, the results of the 2:2 ratio transfection suggest that the effects of disinhibition of cAMP, mediated through the mutant $G$ protein, override the suppressive effect of the native $\mathrm{G}$ protein on cAMP levels.

Residue F200 is highly conserved in a region of $\mathrm{G}$ proteins involved in GTP binding and hydrolysis (21) (Fig. 5). A number of mutations that have been identified in this region affect GTPase and $G$ protein function. For example, in both $G_{\alpha s}$ and $\mathrm{G}_{\alpha \mathrm{i}}$, mutation $\mathrm{Q} \rightarrow \mathrm{L}$ (position 205 in $\mathrm{G}_{\alpha \mathrm{\alpha} 2}$ ) inhibits GTPase activity and causes constitutive activation of the $G$ protein $(8,14$, $23,24)$, whereas mutation of the adjacent G204 $\rightarrow$ A blocks activation by GTP and results in loss of activity (25). X-ray crystallographic studies of $\mathrm{G}_{\alpha \mathrm{i} 1}(26)$ and $\mathrm{G}_{\alpha \mathrm{t}}(27)$ indicate that GTP-binding alters the conformation of three switch regions, among which switch II contains residue F200 (Fig. 5). This conserved residue in $\mathrm{G}_{\alpha \mathrm{t}}$ makes contact with $\mathrm{G}_{\beta \gamma}$ (27). These data, together with our present findings, indicate the importance of residue F200 in the function of $\mathrm{G}_{\alpha \mathrm{i} 2}$.

Somatic cell mutations that affect normal $G$ protein function have been identified in a variety of endocrine tumors $(8$, 23). Our findings suggest that somatic cell mutations in genes of the cAMP-dependent signal transduction pathway may occur at specific sites in the developing myocardium and give rise to a focus with arrhythmogenic potential. In tissues where cells rapidly turn over, somatic cell mutations would be lost unless they occurred in a stem cell or were in some way mitogenic. 


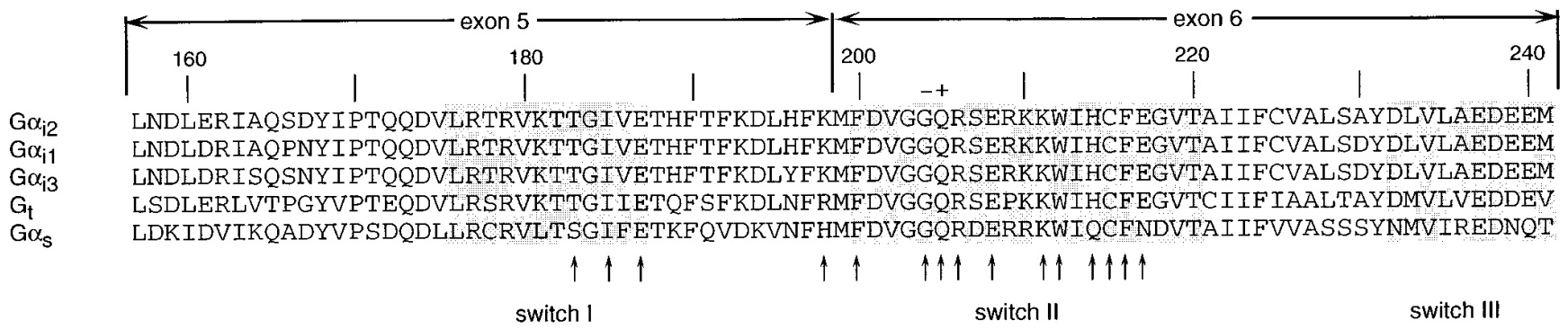

Figure 5. Alignment of sequences from $G$ proteins corresponding to exons 5 and 6 of human $\mathrm{G}_{\alpha i 2}$. Numbering is for amino acid residues in $\mathrm{G}_{\mathrm{\alpha i2} 2} \uparrow$ indicates residues that have been shown to make contacts between $\mathrm{G}_{\mathrm{t}}$ and $\mathrm{G}_{\beta \gamma}(27)$. Shaded areas indicate switch regions that undergo conformational changes upon GTP hydrolysis (27). $(+)$ and $(-)$ indicate the position of activating and inactivating mutations described in the text.

However, in a nondividing cell type a mutation would be fixed in the tissue. Since most data suggest that myocardial cells do not divide (28), a somatic cell mutation would have to occur during embryological development. Myocardial lineage studies in avian embryos using retroviral tagging procedures (2931) have shown that the myocardium is built from cone-shaped growth units that traverse the myocardial wall from epicardial to endocardial surfaces. Each of these growth units is composed of a limited number of clones that arise from relatively few myocardial progenitors in the precardiac mesoderm. Since there is very limited migration of the differentiating cardiomyocytes in the tubular stage heart, the members of each clone do not disperse widely in the myocardium. Thus, a mutation occurring during cardiogenesis would be expected to form a discrete focus, the size of which would represent the embryonic stage at which the mutation occurred. Although the causative mutations occur in utero, their physiological effects might not be manifested until stimulated by some aspect of the hypertrophic mechanisms that mediate postnatal cardiac development, or until degradation of compensatory mechanisms that otherwise suppress the activity of such mutant genes occurs.

It is possible that other somatic mutations in the heart will be subsequently identified, some of which may result in arrhythmogenesis of the conduction system or myocytes. We surmise that somatic mutations of the $\beta$-adrenergic receptoradenylyl cyclase cascade may possibly account for other forms of idiopathic cAMP-mediated ventricular tachycardia.

\section{Acknowledgments}

We are indebted to Dr. Marvin Gershengorn, Dr. Donald Fischman, and Dr. Ralph Nachman for their critical review of the manuscript.

This work was supported in part by grant HL-56139 from the National Institutes of Health. Drs. Lerman and Catanzaro are Established Investigators of the American Heart Association.

\section{References}

1. Belhassen, B., and S. Viskin. 1993. Idiopathic ventricular tachycardia and fibrillation. J. Cardiovasc. Electrophysiol. 4:356-368.

2. Lerman, B.B., L. Belardinelli, G.A. West, R.M. Berne, and J.P. DiMarco. 1986. Adenosine-sensitive ventricular tachycardia: evidence suggesting cyclic AMP-mediated triggered activity. Circulation. 74:270-280.

3. Lerman, B.B. 1993. Response of nonreentrant catecholamine-mediated ventricular tachycardia to endogenous adenosine and acetylcholine. Evidence for myocardial receptor-mediated effects. Circulation. 87:382-390.
4. Lerman, B.B., K.M. Stein, E.D. Engelstein, D. Battkeman, N. Lippman, D. Bei, and D.F. Catanzaro. 1995. Mechanisms of repetitive monomorphic ventricular tachycardia. Circulation. 92:422-429.

5. Song, Y., S. Thedford, B.B. Lerman, and L. Belardinelli. 1992. Adenosine-sensitive afterdepolarizations and triggered activity in guinea pig ventricular myocytes. Circ. Res. 70:743-753.

6. Lerman, B.B., R.C. Wesley, J.P. DiMarco, D.E. Haines, and L. Belardinelli. 1988. Antiadrenergic effects of adenosine on His-Purkinje automaticity: evidence for accentuated antagonism. J. Clin. Invest. 82:2127-2135.

7. Belardinelli, L., and G. Isenberg. 1983. Actions of adenosine and isoproterenol on isolated ventricular myocytes. Circ. Res. 53:287-297.

8. Lyons, J., C.A. Landis, G. Harsh, L. Vallar, K. Grunewald, H. Feichtinger, Q.Y. Duh, O.H. Clark, E. Kawasaki, H.R. Bourne, et al. 1990. Two G protein oncogenes in human endocrine tumors. Science. 249:655-659.

9. Ho, S.N., H.D. Hunt, R.M. Horton, J.K. Pullen, and L.R. Pease. 1989. Site-directed mutagenesis by overlap extension using the polymerase chain reaction. Gene. 77:51-59.

10. Bhattacharya, S., and J. Linden. 1995. The allosteric enhancer, PD 81,723, stabilizes human A1 adenosine receptor coupling to $\mathrm{G}$ proteins. Biochim. Biophys. Acta. 1265:15-21.

11. Kingston, R.E. 1991. Calcium phosphate transfection. In Current Protocols in Molecular Biology. F.M. Ausubel, R. Brent, R.E. Kingston, D.D Moore, J.E. Seidman, J.A. Smith, and K. Struhl, editors. John Wiley and Sons, New York. 9.1.1.

12. Brenner, G.L., K.A. Berg, and M.C. Gershengorn. 1988. Thyroid-stimulating hormone and insulin-like growth factor-1 synergize to elevate 1,2-diacylglycerol in rat thyroid cells. Stimulation of DNA synthesis via interaction between lipid and adenylyl cyclase signal transduction systems. J. Clin. Invest. 82: 1144-1148.

13. Lerman, B.B., K.M. Stein, and S.M. Markowitz. 1996. Idiopathic right ventricular outflow tract tachycardia: a clinical approach. Pace (Pacing Clin. Electrophysiol.). 19:2120-2137.

14. Wong, Y.H., A. Federman, A.M. Pace, I. Zachary, T. Evans, J. Pouyssegur, and H.R. Bourne. 1991. Mutant a subunits of Gi2 inhibit cyclic AMP accumulation. Nature. 351:63-65.

15. Bray, P., A. Carter, V. Guo, C. Puckett, J. Kamholz, A. Spiegel, and M. Nirenberg. 1987. Human cDNA clones for an alpha subunit of Gi signal-transduction protein. Proc. Natl. Acad. Sci. USA. 84:5115-5119.

16. Thomas, J.M., S.R. Meier-Davis, and B.B. Hoffman. 1992. Prolonged activation of inhibitory somatostatin receptors increases adenylate cyclase in wild-type and Gs alpha-deficient (cyc-) S49 mouse lymphoma cells. Cell. Signalling. 4:571-581.

17. Watts, V.J., and K.A. Neve. 1996. Sensitization of endogenous and recombinant adenylyl cyclase by activation of D2 dopamine receptors. Mol. Pharmacol. 50:966-976.

18. Linden, J. 1987. Enhanced cAMP accumulation after termination of cholinergic action in the heart. FASEB J. 1:119-124.

19. Bohm, M., U. Schmidt, P. Gierschik, R.H. Schwinger, S. Bohm, and E. Erdmann. 1994. Sensitization of adenylate cyclase by halothane in human myocardium and S49 lymphoma wild-type and cyc-cells: evidence for inactivation of the inhibitory G protein Gi alpha. Mol. Pharmacol. 45:380-389.

20. Liggett, S.B., M.G. Caron, R.J. Lefkowitz, and M. Hnatowich. 1991. Coupling of a mutated form of the human beta 2-adrenergic receptor to $\mathrm{Gi}$ and Gs. Requirement for multiple cytoplasmic domains in the coupling process. $J$. Biol. Chem. 266:4816-4821.

21. Taussig, R., L.J. Iniguez, and A.G. Gilman. 1993. Inhibition of adenylyl cyclase by Gi alpha. Science. 261:218-221.

22. Taussig, R., W.J. Tang, J.R. Hepler, and A.G. Gilman. 1994. Distinct patterns of bidirectional regulation of mammalian adenylyl cyclases. J. Biol. Chem. 269:6093-6100. 
23. Landis, C.A., S.B. Masters, A. Spada, A.M. Pace, H.R. Bourne, and L. Vallar. 1989. GTPase inhibiting mutations activate the alpha chain of Gs and stimulate adenylyl cyclase in human pituitary tumours. Nature. 340:692-696.

24. Masters, S.B., R.T. Miller, M.H. Chi, F.H. Chang, B. Beiderman, N.G. Lopez, and H.R. Bourne. 1989. Mutations in the GTP-binding site of GS alpha alter stimulation of adenylyl cyclase. J. Biol. Chem. 264:15467-15474.

25. Miller, R.T., S.B. Masters, K.A. Sullivan, B. Beiderman, and H.R. Bourne. 1988. A mutation that prevents GTP-dependent activation of the alpha chain of Gs. Nature. 334:712-715.

26. Mixon, M.B., E. Lee, D.E. Coleman, A.M. Berghuis, A.G. Gilman, and S.R. Sprang. 1995. Tertiary and quaternary structural changes in Gi alpha 1 induced by GTP hydrolysis. Science. 270:954-960.

27. Lambright, D.G., J. Sondek, A. Bohm, N.P. Skiba, H.E. Hamm, and P.B. Sigler. 1996. The 2.0 A crystal structure of a heterotrimeric G protein. $\mathrm{Na}$ ture. 379:311-319.
28. Soonpaa, M.H., and L.J. Field. 1994. Assessment of cardiomyocyte DNA synthesis during hypertrophy in adult mice. Am. J. Physiol. 266:H1439H1445.

29. Mikawa, T., and D.A. Fischman. 1992. Retroviral analysis of cardiac morphogenesis: discontinuous formation of coronary vessels. Proc. Natl. Acad. Sci. USA. 89:9504-9508.

30. Mikawa, T., G.L. Cohen, and D.A. Fischman. 1992. Clonal analysis of cardiac morphogenesis in the chicken embryo using a replication-defective retrovirus. III: polyclonal origin of adjacent ventricular myocytes. Dev. Dyn. 195: 133-141.

31. Mikawa, T., A. Borisov, A.M. Brown, and D.A. Fischman. 1992. Clonal analysis of cardiac morphogenesis in the chicken embryo using a replicationdefective retrovirus: I. Formation of the ventricular myocardium. Dev. Dyn. 193:11-23. 